\title{
Tumores primarios de ámpula de Vater: estadificación prequirúrgica por ultrasonido endoscópico. Estudio de prueba diagnóstica
}

\author{
Ampulary tumors: presurgical staging by endoscopic ultrasonography. Diagnostic test study \\ Alejandro Membrillo-Romero', Mauricio De la Fuente-Lira², Patricia Carvallo-Guevara', \\ Mario E. Rendón-Macías ${ }^{3,4}$ y Alberto Riojas-Garza *

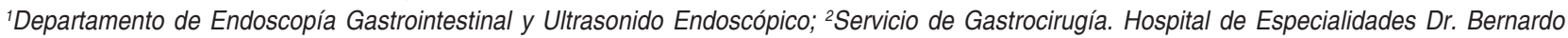 \\ Sepúlveda G., Centro Médico Nacional Siglo XXI, Instituto Mexicano del Seguro Social; ' ${ }^{3}$ nnidad de Investigación en Análisis y Síntesis de la \\ Evidencia, Coordinación de Investigación en Salud, Centro Médico Nacional Siglo XXI, Instituto Mexicano del Seguro Social; ${ }^{4}$ Escuela de Medicina, \\ Universidad Panamericana. Ciudad de México, México
}

\section{Resumen}

Objetivo: Comparar el ultrasonido endoscópico (USE) en la valoración de los tumores ampulares primarios (TAp) con referencia al estudio histopatológico (HP). Método: Estudio retrospectivo prolectivo de pruebas diagnósticas en pacientes con TAp estadificados por USE y pancreatoduodenectomía con estudio HP, atendidos de 2012 a 2018 en un hospital de tercer nivel de atención. Resultados: Fueron incluidos14 pacientes con adenocarcinoma. El tamaño del tumor medido por USE mostró una adecuada correlación $(R=0.65 ; p=0.01)$ con el HP. La exactitud en la determinación de la invasión a la pared duodenal, el páncreas y el conducto biliar fue del 78.5, el 78.5 y el 57.1\%, respectivamente. Por HP hubo tres T1 (21.4\%), dos T2 (14.2\%) y nueve T3 (64.2\%). La exactitud del estadiaje T y N por USE fue del 71.4\% (kappa $=0.50$ ) y del 50\% (kappa $=-0.04)$, respectivamente. Los errores en la estadificación fueron más frecuentes en los pacientes con prótesis biliar. Conclusión: EI USE mostró utilidad en la determinación del tamaño tumoral y de la invasión al duodeno y al páncreas, y más limitada en la determinación de la invasión al conducto biliar por presencia de prótesis. Se observa una sobreestimación del estadiaje $T$ y una subestimación del $\mathrm{N}$. Es necesario mayor capacitación y sugerimos realizarse antes de colocar una prótesis biliar.

PALABRAS CLAVE: Estadificación. Tumor ampular primario. Ultrasonido endoscópico.

\begin{abstract}
Objective: To analyze the accuracy of endoscopic ultrasound (EUS) in the evaluation of ampullary tumors compared to histopathology (HP) staging. Methods: A retrospective diagnostic test study. Patients with ampullary tumors staged by EUS and postpancreatoduodenectomy HP attended during 2012-2018 at tertiary level hospital. Results: 14 patients with adenocarcinoma where included. Tumor size measured by EUS showed an adequate correlation $(R=0.65 ; p=0.01)$ with HP. The accura$c y$ in the evaluation of duodenal wall, pancreatic, and biliary duct invasion was 78.5, 78.5 and $57.1 \%$, respectively. By HP, there were three T1 (21.4\%), two T2 (14.2\%) and nine T3 (64.2\%). The accuracy of $T$ and N staging by EUS was $71.4 \%$ $($ kappa $=0.50)$ and $50 \%($ kappa $=-0.04)$ respectively. There were more staging errors in patients with biliary stent. Conclusion: EUS was useful in estimating tumor size, duodenal wall and pancreatic invasion. Limitation was found in the evaluation of biliary duct invasion, as well as overestimation of $T$ stage and underestimation of $N$ stage. It is necessary an increased effort in ultrasonography training. We suggest EUS testing before biliary stenting.
\end{abstract}

KEY WORDS: Ampullary tumor. Endoscopic ultrasound. Staging.

\author{
Correspondencia: \\ *Alberto Riojas-Garza \\ Augusto Rodin, 473 \\ Col. Insurgentes Mixcoac, Del. Benito Juárez \\ C.P. 03920, Ciudad de México, México \\ E-mail: ariojasg16@gmail.com
}

Fecha de recepción: 18-06-2018

Fecha de aceptación: 24-08-2018

DOI: $10.24875 / C I R U .18000541$
Cir Cir. 2019;87:278-284

Contents available at PubMed www.cirugiaycirujanos.com 


\section{Introducción}

Los tumores periampulares son procesos neoplásicos originados de la cabeza del páncreas, el duodeno, el conducto biliar común distal o el ámpula de Vater. Los tumores ampulares primarios (TAp) son concretamente del complejo ampular ${ }^{1,2}$. Es importante distinguir los TAp del resto de los tumores periampulares por su mejor pronóstico y por ser frecuentemente localizados y detectados en estadios tempranos ${ }^{3}$.

Los TAp pueden ser benignos o malignos. Los adenomas vellosos y tubulovellosos benignos son los más frecuentes, aunque son considerados lesiones premalignas 4 . Por otro lado, el adenocarcinoma es el tipo histológico maligno más frecuente y sus variedades, por orden de frecuencia, son intestinal, pancreatobiliar, adenocarcinoma poco diferenciado, mucinoso-intestinal y papilar invasivo ${ }^{5-8}$.

Estos tumores son relativamente raros, con una incidencia estimada en 3-4 casos por millón ${ }^{5-7}$. Conforman el $7 \%$ de todos los tumores periampulares y el $0.2 \%$ de los tumores del tracto gastrointestinal ${ }^{9}$. Son más frecuentes en los hombres, con una relación $\mathrm{de} \approx 2: 1$, y la edad promedio de los pacientes en el

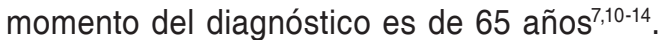

Para la evaluación diagnóstica de los carcinomas ampulares se utilizan instrumentos endoscópicos, radiológicos e histopatológicos ${ }^{2}$. Sin embargo, aunque la colangiopancreatografía retrógrada endoscópica (CPRE) es un estudio útil para el diagnóstico de los tumores ampulares, no permite realizar una adecuada estadificación $n^{2,12}$.

El análisis histopatológico (HP) de la pieza quirúrgica se ha considerado como método de referencia para el estadiaje locorregional de los TAp ${ }^{10,11,13,15-17}$. Actualmente, diversos estudios han valorado la utilidad del ultrasonido endoscópico (USE), el ultrasonido intraductal (USID), la tomografía computarizada y la resonancia magnética para este objetivo. Al momento, el USE y el USID se han considerado los mejores estudios para ese fin ${ }^{10,13,16,18}$.

Independientemente del estadio, la pancreatoduodenectomía es el tratamiento de elección en lesiones malignas invasivas del ámpula de Vater, pues la resección quirúrgica con márgenes negativos es el único tratamiento potencialmente curativo ${ }^{3,19-21}$. Por otro lado, para los adenomas ampulares, la escisión endoscópica o ampulectomía endoscópica ha surgido como un método curativo con buenos resultados ${ }^{22}$. Debido a su carácter poco invasivo, algunos autores ahora han propuesto esta técnica como alternativa para el tratamiento de tumores malignos, sobre todo si se cumplen algunas condiciones, como estadio Tis o T1, tamaño menor de $2 \mathrm{~cm}$ y grado histológico bien diferenciado ${ }^{23,24}$. Sin embargo, el tratamiento de estos tumores es aun controversial debido a la dificultad de una estadificación preoperatoria correcta mediante imagen o endoscopía.

Aunque existen estudios en otras partes del mundo que han analizado el desempeño del USE en el estadiaje locorreginonal de los TAp, en México no se ha reportado información al respecto. Los resultados descritos varían notablemente y existe limitada consistencia. Además, al ser el USE un instrumento dependiente del operador, es necesario contar con evidencia que justifique las actitudes terapéuticas de un centro de salud. Dicha evidencia no existe en nuestro hospital ni en ningún otro centro hospitalario del país.

El objetivo de este estudio fue evaluar el desempeño del USE en la estadificación locorregional de los TAp utilizando como referencia el análisis HP posquirúrgico. A nuestro conocimiento, es el primer estudio en México que evalúa el desempeño de esta prueba para la estadificación de dichos tumores tomando como referencia el estudio HP posquirúrgico.

\section{Método}

Estudio retrospectivo de casos evaluados prolectivamente. Los pacientes incluidos fueron aquellos con sospecha de TAp por estudios previos de CPRE o USE, y enviados para estadifiación por USE, y posible resección quirúrgica del tumor por pancreatoduodenectomía. Los pacientes fueron reclutados del 1 de enero de 2012 al 31 de mayo de 2018. Todos fueron atendidos en el Servicio de Ultrasonido Endoscópico del Hospital de Especialidades del Centro Médico Nacional Siglo XXI. Se excluyeron los pacientes con cirugías abdominales previas que alteraran la visualización por USE o si estaban recibiendo terapia paliativa por enfermedad metastásica. Con el objetivo de tener mayor consistencia en las evaluaciones ultrasonográfica, todos los USE fueron realizados por un solo operador. Así mismo, cuando se realizó una pancreatoduodenectomía, esta fue ejecutada en nuestra unidad por el mismo cirujano. El tiempo entre el USE y la resección quirúrgica tuvo una mediana de 7 días. En todos los pacientes incluidos se obtuvo información sobre su edad y sexo, así como la historia clínica y los estudios de laboratorio previos. 


\section{Ultrasonido endoscópico}

Para este estudio se utilizaron equipos de USE radial (Fujinon EO-53OUT2) y lineal (Fujinon EG580UR), uno u otro dependiendo de la necesidad de toma de biopsia durante el procedimiento. Todos los análisis se realizaron por medio de un abordaje por vía transduodenal y transgástrica. Con el estudio de USE se obtuvo evidencia de la presencia de prótesis biliar, el tamaño tumoral en su diámetro mayor (milímetros) y la condición de invasión del tumor a estructuras vecinas (duodenal, pancreática y ductal). Para la estadificación tumoral se utilizó el sistema TNM ${ }^{25}$. Se consideró la afección exclusivamente ampular cuando la lesión tumoral, identificada en el USE como una masa hipoecoica, crecía hacia la luz y afectaba hasta la submucosa duodenal. La invasión a la pared duodenal se consideró cuando la lesión lograba traspasar la muscular propia de la pared duodenal, donde puede afectar la porción ductal intraampular. La invasión pancreática se consideró cuando la lesión sobrepasaba la pared duodenal y contactaba la glándula pancreática. Para la estadificación con el sistema TNM se definió T1 como una masa hipoecoica con extensión intraampular y hasta la submucosa duodenal; T2 cuando la masa hipoecoica intraampular alcanzaba a contactar la muscular propia duodenal sin sobrepasarla; T3 si existían imágenes compatibles con invasión tumoral a páncreas, colédoco, tejido periduodenal o peripancreático, siempre sobrepasando la muscular propia duodenal; y T4 cuando hubo invasión al eje celiaco, la arteria mesentérica superior o la arteria hepática común. Para el estadio N, se consideró NO en ausencia de imágenes compatibles con invasión a nódulos regionales; $\mathrm{N} 1$ con involucro de uno a tres nódulos regionales; y N2 con cuatro o más nódulos regionales involucrados.

\section{Análisis histopatológico}

El análisis HP se llevó a cabo en las piezas quirúrgicas (pancreatoduodenectomías). Se colocaron en bloques de parafina cortes seriados de las estructuras, y se utilizó tinción de hematoxilina y eosina para el análisis al microscopio.

Se recopilaron los siguientes datos: tipo y grado de diferenciación tumoral, tamaño tumoral macroscópico de su diámetro mayor en milímetros, invasión a estructuras vecinas (duodenal, pancreática y ductal) y estadio ( $\mathrm{T}$ y N).

\section{Análisis estadístico}

Se utilizaron la prueba de ji al cuadrado de $\mathrm{McNe}$ mar (variables dicotómicas), la prueba eta (variable nominal con ordinal) y la prueba t de Student pareada para observar las diferencias en variables continuas y discretas, respectivamente. Así mismo, se determinó el grado de correlación entre la medición del tamaño del tumor por USE y la obtenida de la pieza quirúrgica por medio de correlación de Pearson. La estimación del desempeño del USE se calculó con la exactitud por medio de la concordancia observada en los parámetros de invasión duodenal, pancreática o ductal y estadio ( $\mathrm{T}$ y N); se utilizó el estimador kappa para el estadio ( $\mathrm{T}$ y $\mathrm{N}$ ). Todos los análisis se realizaron con el paquete estadístico SPSS versión 20 y se utilizó un nivel de significancia estadística de $p<0.05$.

\section{Aspectos éticos}

Los procedimientos se realizaron bajo el consentimiento informado firmado de los pacientes como parte de la atención otorgada y de acuerdo con los lineamientos del hospital.

\section{Resultados}

Durante el periodo de enero de 2012 a mayo de 2018 se estadificaron por USE 68 pacientes con TAp. Un gran porcentaje regresó a su ciudad de origen para el manejo médico o quirúrgico $(n=47)$ y otros presentaron enfermedad avanzada con criterios de irresecabilidad $(n=7)$. Solo 14 pacientes fueron sometidos a pancreatoduodenectomía y estadificación tumoral por HP en este hospital, y son los analizados en este estudio (Fig. 1). La edad promedio de los pacientes fue de 63 años y hubo mayor proporción del sexo femenino (70\%). El tipo histológico más común consistió en el adenocarcinoma intestinal bien diferenciado (35.5\%). La mayoría de los estudios endoscópicos se realizaron con ultrasonido lineal (70\%). El $43 \%$ de los pacientes portaban prótesis biliar colocada previamente al USE para el manejo de la ictericia obstructiva (Tabla 1).

En relación con el tamaño tumoral, encontramos una correlación de $R=0.653(p=0.01)$ entre las mediciones obtenidas por USE contra el HP, cuyas mediciones fueron muy semejantes en ambos estudios (media: $23.9 \pm 10$ contra $22.1 \pm 16 \mathrm{~mm}$, respectivamente) (Tabla 2). 


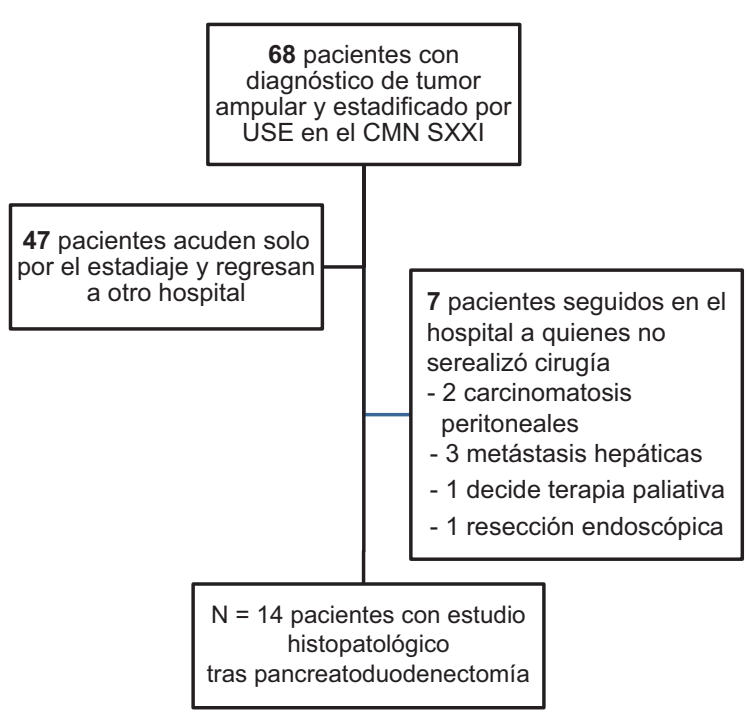

Figura 1. Flujograma de pacientes. CMN SXXI: Centro Médico Nacional Siglo XXI; USE: ultrasonido endoscópico.

Tabla 1. Características basales de los pacientes y de los tumores

\begin{tabular}{lc}
\hline Variable & $\mathbf{N}=14$ \\
\hline Edad en años ( \pm desviación & $63( \pm 12)$ \\
estándar) & \\
Sexo & \\
Femenino & $10(71.4 \%)$ \\
Masculino & $4(28.6 \%)$ \\
Tipo de USE & \\
Lineal & $10(71.4 \%)$ \\
Radial & $4(28.6 \%)$ \\
Prótesis biliar & \\
Sí & $6(42.9 \%)$ \\
No & $8(57.1 \%)$ \\
Tipo histológico & \\
Adenocarcinoma intestinal & \\
Adenocarcinoma pancreatobiliar & $5(35.7 \%)$ \\
Adenocarcinoma papilar & $3(21.4 \%)$ \\
invasivo & $2(14.3 \%)$ \\
Adenocarcinoma no & \\
especificado & $4(28.6 \%)$ \\
Diferenciación tumoral & \\
Bien diferenciado & $9(64.3 \%)$ \\
Moderadamente diferenciado & $5(35.7 \%)$ \\
\hline
\end{tabular}

En cuanto a la invasión de la pared duodenal, de los 14 casos hubo concordancia de invasión en cinco y de no invasión en seis; en los tres restantes con discordancia, dos fueron sobreestadificados por el USE y en uno el USE no detectó la invasión del tumor. Por tanto, la concordancia observada fue del $78.5 \%$ (11/14) (Tabla 3). En la evaluación de la invasión pancreática, la concordancia observada fue del $78.5 \%$ (11/14); en tres pacientes se encontró concordancia de invasión y en ocho de no invasión por ambos estudios. Para los tres casos con discordancia, dos fueron invasiones no detectadas por USE y uno sobreestadificado por USE. Finalmente, con respecto a la invasión ductal, la concordancia observada fue del $57.1 \%$ (8/14), con un acuerdo de invasión en dos casos y de no invasión en seis; la discordancia en los seis restantes fue por infraestadificación en tres y por sobreestadificación en los otros tres.

Al evaluar el estadio $T$ (Tabla 2) de los tumores en el USE no se detectaron T1 ni T4, y estos últimos tampoco fueron identificados por HP. Con ambos métodos, los T3 fueron los más frecuentes (USE 57.1\% y HP $64.2 \%$ ) y no hubo diferencias estadísticamente significativas en la distribución por métodos $(p=0.86)$. De los 14 pacientes analizados, existió concordancia en 10 casos (dos T2 y ocho T3) (71.4\%). La tendencia de este parámetro es a sobreestadificar, pues de los cuatro pacientes mal estadificados tres correspondieron a estadios más tempranos (21.4\%) (T1) y uno a un estadio mayor (7\%) (T3) (Tabla 4). Se estimó el valor kappa para el estadio $\mathrm{T}$, el cual mostró un valor de $0.500(p=0.003)$.

Con respecto al estadio $\mathrm{N}$ de los tumores, existió concordancia en siete casos (cinco No y dos N1) (Tabla 2). La tendencia de este parámetro es a sobreestadificar, pues de los siete pacientes mal estadificados cuatro correspondieron a estadios más tempranos (28\%) (N0) y tres a estadios más tardíos (N1) (21.4\%) (Tabla 5). De igual manera, se estimó el valor kappa para el estadio $\mathrm{N}$ tumoral y fue de $-0.043(p=0.87)$.

\section{Discusión}

Ante el posible beneficio de poder estadificar los tumores ampulares antes de tomar la decisión de un procedimiento quirúrgico muy riesgoso, usar el USE para este propósito ha sido muy alentador; sin embargo, su utilidad aún necesita ser evaluada en nuestras condiciones de trabajo. Por ello, en este estudio se evaluó su desempeño considerando el análisis HP como el método de referencia. Nuestros resultados indican una buena correlación con respecto a la medición del tamaño tumoral ( $R$ de Pearson $=0.653$ ), una exactitud moderada del $78.5,78.5$ y $57.1 \%$ para determinar el grado de invasión duodenal, pancreática y ductal, respectivamente, una exactitud adecuada para el estadio $\mathrm{T}(71.4 \%$, kappa $=0.500)$ y una mala exactitud en la determinación del estadio N (50\%; kappa $=-0.043$ ). 
Tabla 2. Características tumorales por ultrasonido y por estudio histopatológico

\begin{tabular}{|c|c|c|c|}
\hline Variable & USE $(N=14)$ & $\mathrm{HP}(\mathrm{N}=14)$ & p \\
\hline $\begin{array}{l}\text { Tamaño tumoral promedio } \\
\text { (mm } \pm \text { desviación } \\
\text { estándar) }\end{array}$ & $23.9( \pm 10)$ & $22.14( \pm 16)$ & $0.59^{*}$ \\
\hline \multicolumn{4}{|l|}{ Invasión pared duodenal } \\
\hline Sí & $7(50.0 \%)$ & $6(50.0 \%)$ & \multirow[t]{2}{*}{1.00} \\
\hline No & $7(50.0 \%)$ & $8(50.0 \%)$ & \\
\hline \multicolumn{4}{|l|}{ Invasión pancreática } \\
\hline Sí & $4(28.6 \%)$ & $5(35.7 \%)$ & \multirow[t]{2}{*}{1.00} \\
\hline No & $10(71.4 \%)$ & $9(64.3 \%)$ & \\
\hline \multicolumn{4}{|l|}{ Invasión ductal } \\
\hline Sí & $5(35.7 \%)$ & $5(35.7 \%)$ & \multirow[t]{2}{*}{$1.00^{\prime}$} \\
\hline No & $9(64.3 \%)$ & $9(64.3 \%)$ & \\
\hline \multicolumn{4}{|l|}{$\mathrm{T}$} \\
\hline $\mathrm{T} 1$ & $0(0 \%)$ & $3(21.4 \%)$ & \multirow[t]{4}{*}{0.86} \\
\hline $\mathrm{T} 2$ & $6(42.9 \%)$ & $2(14.2 \%)$ & \\
\hline T3 & $8(57.1 \%)$ & $9(64.2 \%)$ & \\
\hline $\mathrm{T} 4$ & $0(0 \%)$ & $0(0 \%)$ & \\
\hline \multicolumn{4}{|l|}{$N$} \\
\hline NO & $8(57.1 \%)$ & $9(64.3 \%)$ & \multirow[t]{2}{*}{$1.00^{\prime}$} \\
\hline N1 & $6(42.9 \%)$ & $5(35.7 \%)$ & \\
\hline
\end{tabular}

*Prueba t de Student pareada. †Prueba de ji al cuadrado de McNemar. "Prueba de eta. Valor de P para prueba t de Student pareada y prueba exacta de Fisher. HP: histopatología; N: nódulos; T: tamaño; USE: ultrasonido endoscópico.

Tabla 3. Relación entre la invasión duodenal, pancreática y ductal por ultrasonido y por estudio histopatológico

\begin{tabular}{lccc}
\hline \multirow{2}{*}{$\begin{array}{l}\text { Invasión } \\
\text { duodenal }\end{array}$} & \multicolumn{3}{c}{ HP } \\
\cline { 2 - 4 } & Sí & No & Total \\
\hline USE & & 2 & 7 \\
Sí & 5 & 6 & 7 \\
No & 1 & 8 & 14 \\
Total & 6 & $75 \%$ & $78.5 \%$ \\
Exactitud & $83.3 \%$ & &
\end{tabular}

Invasión páncreas

$\begin{array}{lccc}\text { USE } & 3 & 1 & 4 \\ \text { Sí } & 2 & 8 & 10 \\ \text { No } & 5 & 9 & 14 \\ \text { Total } & 60 \% & 88.8 \% & 78.5 \% \\ \text { Exactitud } & & & \end{array}$

Invasión ductal

\begin{tabular}{lccc} 
USE & 2 & 3 & 5 \\
Sí & 3 & 6 & 9 \\
No & 5 & 9 & 14 \\
Total & $40 \%$ & $66.6 \%$ & $57.1 \%$ \\
Exactitud & & \\
\hline HP: histopatología: USE: ultrasonido endoscópico. &
\end{tabular}

HP: histopatología; USE: ultrasonido endoscópico.

Tal como ha sido informado en otros estudios, los tamaños tumorales medidos por USE y HP son muy similares ${ }^{17,26}$, y por tanto podemos afirmar que es factible estimar el tamaño real del tumor por medio del
Tabla 4. Relación entre el estadio T por ultrasonido y por estudio histopatológico

\begin{tabular}{lcccc}
\hline & \multicolumn{5}{c}{ HP } \\
\cline { 2 - 5 } & T1 & T2 & T3 & Total \\
\hline USE & & & & \\
T1 & 0 & 0 & 0 & 0 \\
T2 & 3 & 2 & 1 & 6 \\
T3 & 0 & 0 & 8 & 8 \\
T4 & 0 & 0 & 0 & 0 \\
Total & 3 & 2 & 9 & 14 \\
Exactitud & $0 \%$ & $100 \%$ & $88.8 \%$ & $71.4 \%$ \\
\hline HP: histopatología; & USE: ultrasonido endoscópico. & &
\end{tabular}

Tabla 5. Relación entre el estadio $\mathrm{N}$ por ultrasonido y por estudio histopatológico

\begin{tabular}{lccc}
\hline & \multicolumn{3}{c}{ HP } \\
\cline { 2 - 4 } & N0 & N1 & Total \\
\hline USE & & & \\
N0 & 5 & 3 & 8 \\
N1 & 4 & 2 & 6 \\
Total & 9 & 5 & 14 \\
Exactitud & $55.5 \%$ & $40 \%$ & $50 \%$ \\
\hline
\end{tabular}

HP: histopatología; USE: ultrasonido endoscópico.

USE. Esta alta correlación permite usar la medición del USE para apoyar en gran parte el plan quirúrgico en estos pacientes.

En cuanto a la exactitud del USE para la detección de invasión tumoral a estructuras vecinas, como la pared duodenal, el páncreas y los conductos biliares intrapancreáticos, en otras partes del mundo se han reportado valores tan altos como del $90 \%$ para cada parámetro ${ }^{11,12,26}$. Nosotros encontramos una exactitud menor, en particular para la invasión ductal. Estudios previos han señalado que la presencia de una prótesis biliar puede dificultar seriamente la estadificación del tumor por USE para invasión ductal ${ }^{27-29}$. Es de hacer notar que seis pacientes $(40 \%)$ tenían esta condición, y en tres de ellos se observó una clasificación menos exacta, pues en nuestro medio, al ser un hospital de referencia con tiempos de atención muchas veces largos, a los pacientes se les trata la ictericia obstructiva potencialmente letal antes de enviarlos a su estadificación. Por otro lado, la sobreestadificación de la extensión puede ser atribuida al edema inflamatorio peritumoral, debido a las características del tumor o a su manipulación cuando se coloca la prótesis. Estas imágenes pueden confundir la expansión tumoral ${ }^{30}$. Otra causa pudiera ser la necesidad de mayor capacitación. Tal error de sobreestadificación implica una frecuencia más alta 
de pancreatoduodenectomías, lo cual podría impactar en la morbilidad; sin embargo, puede existir a su vez una mayor tasa de éxito quirúrgico en relación con la resección total tumoral. Una mayor exactitud observamos en la evaluación de la invasión a la pared duodenal y el páncreas. Esta invasión suele mostrar imágenes más nítidas y por tanto fáciles de detectar, además de la adecuada capacidad del USE para identificar la capa muscular duodenal.

La exactitud calculada en nuestro estudio para el estadio T tumoral $(71.4 \%)$ se encuentra dentro de los valores reportados en la literatura $(60-90 \%)^{10-13,16-18,26}$. En nuestros pacientes, el uso del USE no nos permitió detectar los tumores T1, pues los tres pacientes en esta condición fueron sobreestadificados e identificados como tumores T2. Todos tenían tumores polipoides con edema en su sitio de implantación, lo cual pudo ser la causa de la sobreestadificación. Son necesarios más estudios para confirmar esta situación. La mayor exactitud se encontró al estadificar los tumores T3 (88.8\%), tal como ha sido encontrado por otros autores ${ }^{11,16-18}$, pues la masa tumoral es mayor y más evidente con cualquier método de evaluación. Cabe destacar que, en nuestra serie, solo un caso fue infraestadificado. Otros estudios han reportado valores kappa de 0.51 a 0.56 para el estadio $\mathrm{T}^{10,17}$, pero en este trabajo el estimado se encuentra ligeramente por debajo (0.500). Como ya se mencionó, el grado de edema perilesional está relacionado con la sobreestimación de los tumores. Sería necesario concentrar la atención en la distinción de la inflamación y la expansión tumoral para una mejor estadificación por USE y, en definitiva, su realización previamente a cualquier manipulación tumoral.

En cuanto a la exactitud calculada para el estadio tumoral N (50\%), la encontramos por debajo de lo informado en otros estudios ${ }^{10,11,13,17,18}$ que la estiman en un $60-80 \%$. Los criterios para calificar a un nódulo linfático como metastásico por USE (bordes regulares, márgenes marcados, homogeneidad hipoecoica, $<2 \mathrm{~cm}$ ) se presentan en solo el $25 \%$ de los ganglios con metástasis; además, dos de estos criterios pueden ser un hallazgo de ganglios normales ${ }^{28,31}$. Para poder corroborar el estado de los ganglios y lograr diferenciarlos de una adenopatía inflamatoria es necesaria la biopsia ganglionar, lo cual incrementaría los costos exponencialmente.

Varias limitaciones deben ser consideradas en nuestro estudio. La primera es el tamaño de la muestra, que fue relativamente pequeño, pues se sabe que la incidencia de esta patología es baja en nuestro medio y en todo el mundo. Esto no permitió la evaluación de la precisión de nuestros estimados. Por último, el resultado de patología dependió del patólogo en turno y no de un consenso en el servicio, lo cual podría modificar el grado de confiabilidad en relación con la estadificación de estos estudios.

\section{Conclusiones}

EI USE es una herramienta no invasiva que parece permitir una medición adecuada de los tumores ampulares. Se recomienda continuar la capacitación y prestar mayor atención en la distinción del tumor y el edema perilesional, así como un análisis previo a la colocación de una prótesis biliar o a la manipulación tumoral. Para una mejor estadificación podría ser necesaria la biopsia de los ganglios identificados. Es necesario realizar estudios con mayor tamaño de muestra para mejorar la precisión de los resultados.

\section{Conflicto de intereses}

Los autores declaran no tener conflicto de intereses.

\section{Financiamiento}

El presente trabajo no obtuvo financiamiento en ninguna forma.

\section{Responsabilidades éticas}

Protección de personas y animales. Los autores declaran que para esta investigación no se han realizado experimentos en seres humanos ni en animales.

Confidencialidad de los datos. Los autores declaran que han seguido los protocolos de su centro de trabajo sobre la publicación de datos de pacientes.

Derecho a la privacidad y consentimiento informado. Los autores han obtenido el consentimiento informado de los pacientes y/o sujetos referidos en el artículo. Este documento obra en poder del autor de correspondencia.

\section{Bibliografía}

1. Ahn DH, Bekaii-Saab T. Ampullary cancer: an overview. American Society of Clinical Oncology educational book. American Society of Clinical Oncology Meeting; 2014. p. 112-5.

2. Martin JA. Ampullary carcinoma: Epidemiology, clinical manifestations, diagnosis and staging. UpToDate. Disponible en: https://www.uptodate. com/contents/ampullary-carcinoma-epidemiology-clinical-manifestations-diagnosis-and-staging Ultimo acceso: 09 de marzo 2019.

3. Ryan DP, Mamon H, Fernandez-del Castillo C. Ampullary carcinoma: treatment and prognosis. Disponible en: https://www.uptodate.com/contents/ampullary-carcinoma-treatment-and-prognosis . Ultimo acceso: 15 de marzo 2019 
4. Takashima M, Ueki T, Nagai E, Yao T, Yamaguchi K, Tanaka M, et al. Carcinoma of the ampulla of Vater associated with or without adenoma: a clinicopathologic analysis of 198 cases with reference to p53 and Ki-67 immunohistochemical expressions. Mod Pathol. 2000; 13:1300-7.

5. Albores-Saavedra J, Schwartz AM, Batich K, Henson DE. Cancers of the ampulla of vater: demographics, morphology, and survival based on 5,625 cases from the SEER program. J Surg Oncol. 2009; 100:598-605.

6. Koea J, Phillips A, Lawes C, Rodgers M, Windsor J, McCall J. Gall bladder cancer, extrahepatic bile duct cancer and ampullary carcinoma in New Zealand: demographics, pathology and survival. ANZ J Surg. 2002;72:857-61.

7. Renard P, Boutron MC, Faivre J, Milan C, Bedenne L, Hillon P, et al Biliary tract cancers in Cote-d'Or (France): incidence and natural history. J Epidemiol Commun Health. 1987;41:344-8.

8. Ruemmele P, Dietmaier W, Terracciano L, Tornillo L, Bataille F, Kaiser $A$, et al. Histopathologic features and microsatellite instability of cancers of the papilla of Vater and their precursor lesions. Am J Surg Pathol. 2009;33:691-704.

9. Jemal A, Siegel R, Ward E, Hao Y, Xu J, Murray T, et al. CA Cancer J Clin. 2008:58:71-96.

10. Artifon EL, Couto D, Jr., Sakai P, da Silveira EB. Prospective evaluation of EUS versus CT scan for staging of ampullary cancer. Gastrointest Endosc. 2009;70:290-6.

11. Kubo $H$, Chijiiwa $Y$, Akahoshi $K$, Hamada S, Matsui N, Nawata $H$. Pre-operative staging of ampullary tumours by endoscopic ultrasound. Br J Radiol. 1999;72:443-7.

12. Ridtitid W, Schmidt SE, Al-Haddad MA, LeBlanc J, DeWitt JM, McHenry $\mathrm{L}$, et al. Performance characteristics of EUS for locoregional evaluation of ampullary lesions. Gastrointest Endosc. 2015;81:380-8.

13. Skordilis P, Mouzas IA, Dimoulios PD, Alexandrakis G, Moschandrea J, Kouroumalis $\mathrm{E}$. Is endosonography an effective method for detection and local staging of the ampullary carcinoma? A prospective study. BMC Surg. 2002;2:1.

14. Talamini MA, Moesinger RC, Pitt HA, Sohn TA, Hruban RH, Lillemoe $\mathrm{KD}$, et al. Adenocarcinoma of the ampulla of Vater. A 28-year experience. Ann Surg. 1997;225:590-9; discussion 599-600.

15. Chen $\mathrm{CH}$, Tseng LJ, Yang CC, Yeh YH, Mo LR. The accuracy of endoscopic ultrasound, endoscopic retrograde cholangiopancreatography, computed tomography, and transabdominal ultrasound in the detection and staging of primary ampullary tumors. Hepatogastroenterology. 2001;48:1750-3.

16. Ito K, Fujita N, Noda Y, Kobayashi G, Horaguchi J, Takasawa O, et al. Preoperative evaluation of ampullary neoplasm with EUS and transpapillary intraductal US: a prospective and histopathologically controlled study. Gastrointest Endosc. 2007;66:740-7.
17. Wee E, Lakhtakia S, Gupta R, Anuradha S, Shetty M, Kalapala R, et al. The diagnostic accuracy and strength of agreement between endoscopic ultrasound and histopathology in the staging of ampullary tumors. Indian J Gastroenterol. 2012;31:324-32.

18. Chen $\mathrm{CH}$, Yang $\mathrm{CC}$, Yeh $\mathrm{YH}$, Chou DA, Nien CK. Reappraisal of endosonography of ampullary tumors: correlation with transabdominal sonography, CT, and MRI. J Clin Ultrasound. 2009;37:18-25.

19. Beger HG, Treitschke F, Gansauge F, Harada N, Hiki N, Mattfeldt T. Tumor of the ampulla of Vater: experience with local or radical resection in 171 consecutively treated patients. Arch Surg. 1999;134:526-32.

20. Di Giorgio A, Alfieri S, Rotondi F, Prete F, Di Miceli D, Ridolfini MP, et al. Pancreatoduodenectomy for tumors of Vater's ampulla: report on 94 consecutive patients. World J Surg. 2005;29:513-8.

21. Paramythiotis D, Kleeff J, Wirtz M, Friess $H$, Buchler MW. Still any role for transduodenal local excision in tumors of the papilla of Vater? J Hepatobiliary Pancreat Surg. 2004;11:239-44.

22. Ceppa EP, Burbridge RA, Rialon KL, Omotosho PA, Emick D, Jowell PS, et al. Endoscopic versus surgical ampullectomy: an algorithm to treat disease of the ampulla of Vater. Ann Surg. 2013:257:315-22.

23. Neves P, Leitao M, Portela F, Pontes JM, Areia M, Brito D, et al. Endoscopic resection of ampullary carcinoma. Endoscopy. 2006;38:101.

24. Woo SM, Ryu JK, Lee SH, Lee WJ, Hwang JH, Yoo JW, et al. Feasibility of endoscopic papillectomy in early stage ampulla of Vater cancer. J Gastroenterol Hepatol. 2009;24:120-4.

25. Amin MB, Greene FL, Edge SB, Compton CC, Gershenwald JE, Brookland RK, et al. The Eighth Edition AJCC Cancer Staging Manual: Continuing to build a bridge from a population-based to a more "personalized" approach to cancer staging. CA Cancer J Clin. 2017;67:99-93.

26. Yasuda $K$, Mukai $H$, Cho $E$, Nakajima M, Kawai $K$. The use of endoscopic ultrasonography in the diagnosis and staging of carcinoma of the papilla of Vater. Endoscopy. 1988;20(Suppl 1):218-22.

27. Cannon ME, Carpenter SL, Elta GH, Nostrant TT, Kochman ML, Ginsberg GG, et al. EUS compared with $\mathrm{CT}$, magnetic resonance imaging, and angiography and the influence of biliary stenting on staging accuracy of ampullary neoplasms. Gastrointest Endosc. 1999;50:27-33.

28. Castillo C. Endoscopic ultrasound in the papilla and the periampullary region. World J Gastrointest Endosc. 2010;2:278-87.

29. Fusaroli $\mathrm{P}$, Manta R, Fedeli $\mathrm{P}$, Maltoni S, Grillo A, Giovannini E, et al. The influence of endoscopic biliary stents on the accuracy of endoscopic ultrasound for pancreatic head cancer staging. Endoscopy. 2007;39:813-7.

30. Mortensen MB, Edwin B, Hunerbein M, Liedman B, Nielsen HO, Hovendal C. Impact of endoscopic ultrasonography (EUS) on surgical decision-making in upper gastrointestinal tract cancer: an international multicenter study. Surg Endosc. 2007;21:431-8.

31. Catalano MF, Sivak MV, Jr., Rice T, Gragg LA, Van Dam J. Endosonographic features predictive of lymph node metastasis. Gastrointest Endosc. 1994:40:442-6. 